\title{
Interaction of Airspace Partitions and Traffic Flow Management Delay
}

\author{
Kee Palopo* \\ NASA Ames Research Center, Moffett Field, CA, 94035, USA \\ Gano B. Chatterji ${ }^{\dagger}$ and Hak-Tae Lee ${ }^{\ddagger}$ \\ University of California Santa Cruz, Moffett Field, CA, 94035, USA
}

\begin{abstract}
To ensure that air traffic demand does not exceed airport and airspace capacities, traffic management restrictions, such as delaying aircraft on the ground, assigning them different routes and metering them in the airspace, are implemented. To reduce the delays resulting from these restrictions, revising the partitioning of airspace has been proposed to distribute capacity to yield a more efficient airspace configuration. The capacity of an airspace partition, commonly referred to as a sector, is limited by the number of flights that an air traffic controller can safely manage within the sector. Where viable, re-partitioning of the airspace distributes the flights over more efficient sectors and reduces individual sector demand. This increases the overall airspace efficiency, but requires additional resources in some sectors in terms of controllers and equipment, which is undesirable. This study examines the tradeoff of the number of sectors designed for a specified amount of traffic in a clear-weather day and the delays needed for accommodating the traffic demand. Results show that most of the delays are caused by airport arrival and departure capacity constraints. Some delays caused by airspace capacity constraints can be eliminated by re-partitioning the airspace. Analyses show that about 360 high-altitude sectors, which are approximately today's operational number of sectors of 373, are adequate for delays to be driven solely by airport capacity constraints for the current daily air traffic demand. For a marginal increase of 15 seconds of average delay, the number of sectors can be reduced to 283 . In addition, simulations of traffic growths of $15 \%$ and $20 \%$ with forecasted airport capacities in the years 2018 and 2025 show that delays will continue to be governed by airport capacities. In clear-weather days, for small increases in traffic demand, increasing sector capacities will have almost no effect on delays.
\end{abstract}

\section{Introduction}

$\mathrm{M}$ OST air traffic delays on a given day are a function of the available airport and airspace capacities. Airport arrival and departure capacities depend on the number of available runways, separation requirements, wind and weather conditions. Airspace capacity in the absence of weather is limited mostly by air traffic controller workload considerations. When traffic demand is expected to exceed capacity, traffic flow management techniques, ${ }^{1-7}$ such as delaying fights on the ground, spacing them in the air and changing their routes of flight, are used to curtail demand. The delays cost the airlines and the flying public.

To reduce these delays, ways for increasing airspace efficiency by using under-utilized controller resources rather than reducing demand has been proposed in Ref. 8. This has motivated the development of several algorithms for increasing and distributing capacity by re-partitioning of airspace into more regions of airspace, commonly referred to as sectors. ${ }^{9-14}$ Sectors are sub-divisions of areas of any of the 20 centers in the entire United States airspace within which air traffic controllers are responsible for aircraft separation. More sectors, however, come at the cost of additional air traffic controllers and equipment for operating those sectors. Both traffic flow management techniques and airspace partitioning methods impose a cost on airlines and airspace service provider, respectively.

Most of the past research has focused individually on traffic flow management or airspace partitioning. Two of the more recent research has examined the integration of the two. However, the interaction problems between the two are not yet fully understood. Previous studies that have examined traffic flow decisions in conjunction with airspace partitioning are described in Refs. 15 and 16. The approach in Ref. 15 consists of using forecasted traffic to re-partition

\footnotetext{
*Aerospace Engineer, Systems Modeling and Optimization Branch, M/S 210-15, Member.

${ }^{\dagger}$ Scientist and Task Manager, U. C. Santa Cruz, M/S 210-8, Associate Fellow.

$¥$ Associate Scientist, U. C. Santa Cruz, M/S 210-8, Member.
} 
the Chicago Center airspace into the same number of sectors as in the current system. Capacities of the sectors were set to $5 / 3$ times the flight transit-times in minutes. ${ }^{17}$ Flights were then delayed based on the sector capacity. Authors concluded that re-partitioning of airspace in one center has a system-wide benefit to other centers. They found that less traffic flow management delay is needed for reducing congestion in other centers with the re-partitioned Chicago Center. The Chicago Center itself experiences improved performance due to airspace re-partitioning.

The procedure discussed in Ref. 16 consists of computing routes for avoiding regions of severe weather and ground and airborne delays ${ }^{18}$ for complying with sector and airport capacities. In addition, the airspace is partitioned into sectors. The process of traffic flow management and airspace partitioning is repeated in a cyclical manner. Their preliminary results in delays showed that the process did not converge. Delays go up and down from iteration to iteration.

Based on lessons learned, a data-driven two-step hierarchical approach was developed to study the interaction between airspace partitioning and traffic flow management delay in the absence of weather. In the first step, air traffic position data are forecasted without consideration of airport and airspace capacity constraints using flight-plan data. These data are then provided as input to the method described in Ref. 19 to partition the airspace within the 20 centers in the United States. The second step consists of using the partitioned airspace and the simulated air traffic data from the previous step to determine the sector entry and exit times for each flight. These times, and sector and airport capacities are then provided to a departure scheduler that computes departure times to comply with the airport and airspace capacity constraints.

Results with this approach for current and increased traffic demand along with current and forecasted airport capacities show that 1) airspace can be re-partitioned to eliminate airspace capacity delays, 2) delays are and will continue to be mostly due to airport capacity constraints, which agrees with the conclusions in Refs. 20-22.

The rest of the paper is organized as follows. Section II describes the airspace partitioning, simulation tools, and a departure scheduler used in this study. The two-step hierarchical method is described in Section III. Results of the study are presented in Section IV. Finally, the main findings are summarized in Section V.

\section{Airspace Partition, Simulation Tools and Departure Scheduler}

This section provides a summary of three of the tools used for generating the results of this study. The Voronoidiagram-based sector partition method used for partitioning the airspace into sectors is summarized in Section II.A. A brief description of the Airspace Concept Evaluation System (ACES) that was used for simulating the air traffic is provided in Section II.B. The first-come first-served (FCFS) scheduler for computing departure times needed for complying with the airport and airspace constraints is discussed in Section II.C. Section III describes how these three tools are used together.

\section{A. Voronoi-diagram-based Sector Partitioning Method and Example Partition}

This section provides an overview of the airspace partitioning method and how the method is applied along with an example partition produced. The method recursively uses a Voronoi diagram to optimize airspace partitions. Optimization in the partitioning process is guided by primary and secondary cost functions, and constraints. The components of the primary cost function include average flight-time through the sector, proximity of sector boundary to major flows and crossings of major flows, penalty for number of flights with short flight-time through the sector, and difference between the maximum number of aircraft in a sector compared to other sectors. The last component attempts to create air traffic demand balanced sectors. The secondary cost function is the crossing-angle between the flight paths and the sector boundary. It is desirable to have sector boundaries that do not cross flows, and if they do, they cross at near right angles. This secondary cost function is used as the basis for judging the goodness of the partitions created with the primary cost function.

In addition to these primary and secondary cost functions, two types of constraints have been considered in the algorithm. The first stipulates that capacity of the sector - following the 5/3 transit-time rule - be greater than or equal to the maximum number of aircraft in the sector. The second type of constraint requires that the maximum number of aircraft in the sector at any time be at or below the specified capacity value. Airspace partitions created in this study are based on only the second type of constraint. For further detail on this method, refer to Ref. 19.

The sequential partitioning process consists of several recursive steps. In the first step, the airspace is partitioned in each of the 20 centers into one to six regions horizontally and into two to three regions vertically based on the primary cost function. In the second step, the secondary cost function and the constraints are then used to determine if these sectors need to be partitioned further in the second step. The selected sectors are partitioned further using step one. 
Partitioning is halted once all the sectors meet the goodness criterion of the secondary cost function and satisfy the constraints.

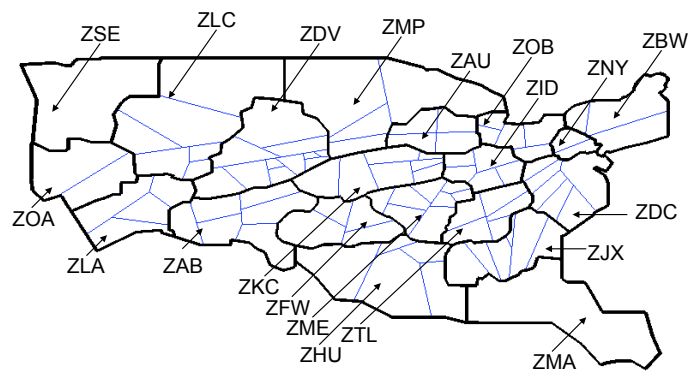

(a) Twenty centers partitioned into sectors at one flight level.

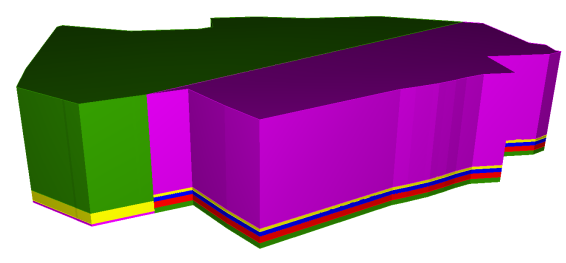

(b) Vertical partitions of the Oakland Center airspace in high altitude.

Figure 1. Example of airspace partitioning.

Figure 1 shows an example of airspace partitions obtained using the procedure described above. Figure 1 (a) shows a horizontal slice of the sectors at a single 24,000 feet altitude level. Figure 1 (b) shows the vertical partitions of the Oakland Center airspace. The number of sectors in each center is summarized in Table 1. This example shows a total of 283 sectors at altitudes 24,000 feet and above generated for the target design capacity of 21 aircraft per sector. Although the Oakland center is shown with two sectors in the horizontal slice, the center has vertical partitions as well as shown in Fig. 1 (b) for a total of eight generated sectors. Similarly, the Seattle center is shown with one sector in the horizontal slice, but the center has five generated sectors as shown in Table 1.

For comparison, the corresponding number of high- and super-high-altitude sectors that serviced the traffic on May 3, 2007 was 373, and each sector had an average capacity of around 17 with a range from 9 to 23 . Also for comparison, the total capacity for the 283 sectors is 5943 and for the May 3rd sectors is 6298.

\section{B. Airspace Concept Evaluation System}

This section provides an overview of ACES and how it is used. ACES is a gate-to-gate simulation of air traffic at local, regional and national levels. It was developed at the NASA Ames Research Center. ${ }^{23}$ ACES simulates flight trajectories using aircraft models derived from the EUROCONTROL's Base of Aircraft Data (BADA) ${ }^{24}$ and traffic data consisting of departure times and flight-plans obtained from the recorded Airline Situation Display to Industry (ASDI) files. Traffic flow management and air traffic control models in ACES use airport and sector capacity thresholds for delaying flights on the ground and in flight. ACES can also be run without traffic flow management. This capability enables one to simulate traffic without capacity constraints as is done in this study.

Typical ACES outputs are arrival and departure counts at airports, traffic-counts in sectors and system performance metrics including arrival, departure, en-route and total delays. Validation studies in Refs. 25 and 26 have shown that ACES generates delays and metrics similar to those observed in the real-world.

In this study, ACES was used for simulating traffic without airport and airspace capacity constraints. The resulting output data were then used for generating inputs for the departure scheduler, which is discussed in the next subsection, and for verifying that the departure schedule generated by the departure scheduler satisfied the capacity constraints. 
Table 1. Number of sectors in the 20 centers

\begin{tabular}{llrr}
\hline \hline \multirow{2}{*}{ Center } & \multirow{2}{*}{ ID } & \multicolumn{2}{c}{ Number of sectors } \\
\cline { 3 - 4 } & & May 3, 2007 & Voronoi \\
\hline Albuquerque & ZAB & 23 & 22 \\
Chicago & ZAU & 25 & 16 \\
Boston & ZBW & 14 & 3 \\
Washington & ZDC & 20 & 18 \\
Denver & ZDV & 29 & 26 \\
Fort Worth & ZFW & 18 & 13 \\
Houston & ZHU & 17 & 12 \\
Indianapolis & ZID & 25 & 19 \\
Jacksonville & ZJX & 24 & 17 \\
Kansas City & ZKC & 27 & 20 \\
Los Angeles & ZLA & 16 & 13 \\
Salt Lake City & ZLC & 8 & 14 \\
Miami & ZMA & 6 & 3 \\
Memphis & ZME & 21 & 20 \\
Minneapolis & ZMP & 18 & 13 \\
New York & ZNY & 8 & 6 \\
Oakland & ZOA & 10 & 8 \\
Cleveland & ZOB & 28 & 17 \\
Seattle & ZSE & 10 & 5 \\
Atlanta & ZTL & 22 & 18 \\
\hline \hline
\end{tabular}

\section{Departure Scheduler}

The departure scheduler uses the FCFS principle to create a departure schedule for all flights. The principle along with its inputs and outputs are overviewed by an example below. The example is for a single flight UAL123 flying through three sectors $\mathrm{Z} 1$ to $\mathrm{Z} 3$.

Inputs to the scheduler are the flight-plan departure time, sector entry and exit times, and arrival time at the destination airport. The scheduler also needs airport arrival/departure capacities and sector capacities.

The FCFS algorithm sorts all flights according to their flight-plan departure times and then begins scheduling by allocating the airport and sector resources to the flights. As flights occupy these resources for the time periods based on their transit-time, the available capacity is reduced to the point that none is available. Flights slated for later departure are delayed to find time intervals when airport and sector capacities are available.

For example, consider scheduling an aircraft with a given transit time for sector Z1 in Fig. 2. Due to all of the previously scheduled flights, Z1 has available capacity during time periods marked "open time interval." The example flight UAL123 can be scheduled such that its transit time interval is within an open time interval past its original/unimpeded scheduled entry time. A time shift with respect to the original/unimpeded scheduled entry time that places the flight completely inside an open interval is called a feasible delay solution. In Fig. 2, green bars are examples of feasible delay solutions while red bars that have overlapping portions with closed time intervals indicate capacity violation. The entire range of feasible delay solution is marked as two gray regions at the bottom of the figure.

Now consider the same example flight passing through multiple sectors from its origin to destination. Fig. 3 illustrates an example of the scheduling procedure with three sectors, Z1 through Z3. Feasible delays are computed for each sector. Common time intervals from the feasible delays across the sequence of sectors are then computed as shown in the figure. Any amount of delay that falls into this common time interval will produce a schedule that satisfies all the capacity constraints. In the FCFS scheduler, the smallest time from the common feasible delays is used, since it is the minimum delay for the flight assuming all the previously assigned schedules are fixed. Once delays are computed for every flight, a new departure time is computed for each flight by adding these delays to the flight-plan departure times.

Although the departure scheduling method was described for sector constraints, the same method is used for airport 
arrival and departure capacity constraints.

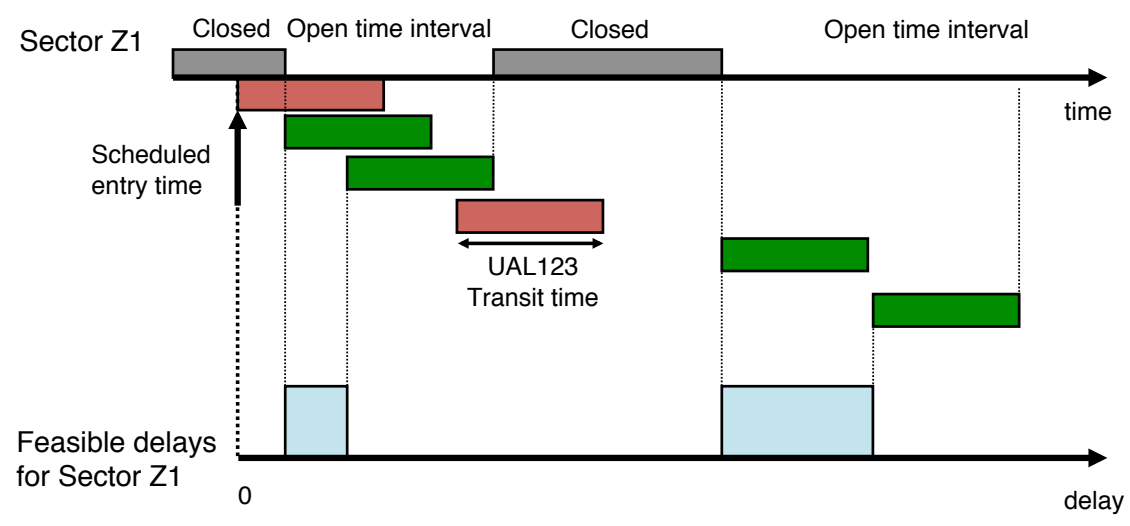

Figure 2. Feasible delay solutions for a flight inside open time-intervals.

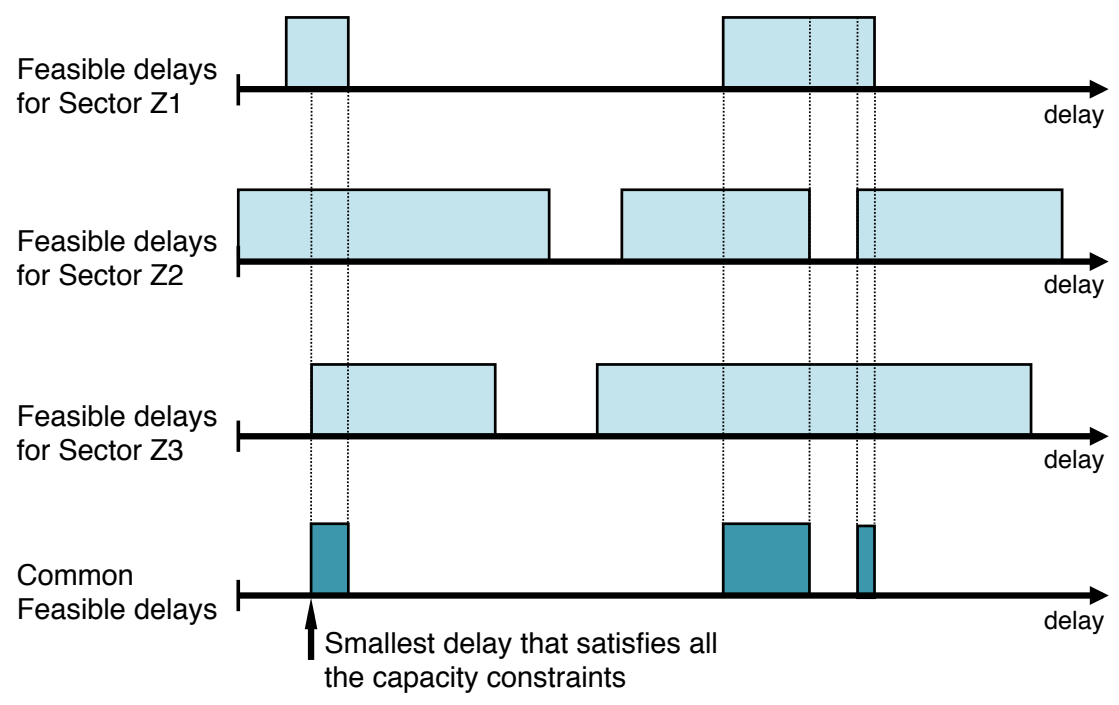

Figure 3. Common feasible delays.

\section{Approach}

This section describes our approach in using all these tools to generate the interaction between the number of sectors and the corresponding delays. The interaction is studied by following the two-step hierarchical method outlined in Fig. 4. The method consists of simulating traffic using ACES, partitioning the airspace using the Voronoi-diagrambased sector partition method, and computing departure schedules with the FCFS departure scheduler. Ten airspace partition sets generated by the sector partition method are presented. The partition sets are used as an input to generate transit times for the scheduler. This study did not consider weather. In follow-up studies, the effect of weather will be explored.

Prior to ACES simulation, the initial input preparation consists of reading a recorded ASDI data file, converting the data to ACES format and writing it out to a flight plan file. The ASDI data consists of twenty-eight hours of "clear" weather-day air-traffic flight plan from May 3, 2007. The flight plan contains aircraft identification, aircraft type, departure airport, arrival airport, scheduled departure time, cruise speed, cruise altitude, and a sequence of points marked by latitudes and longitudes along the route of flight for all flights in the input ASDI file.

Next, air traffic is simulated in Block 1 without traffic flow management using the flight plan file as an input. Without traffic flow management in the simulation, aircraft count is not constrained in any sectors. Similarly, aircraft departure rates and arrival rates at airports are not constrained. This unconstrained capacities allow full traffic demand to be accounted. The aircraft position time history resulting from simulation without capacity constraints is written 


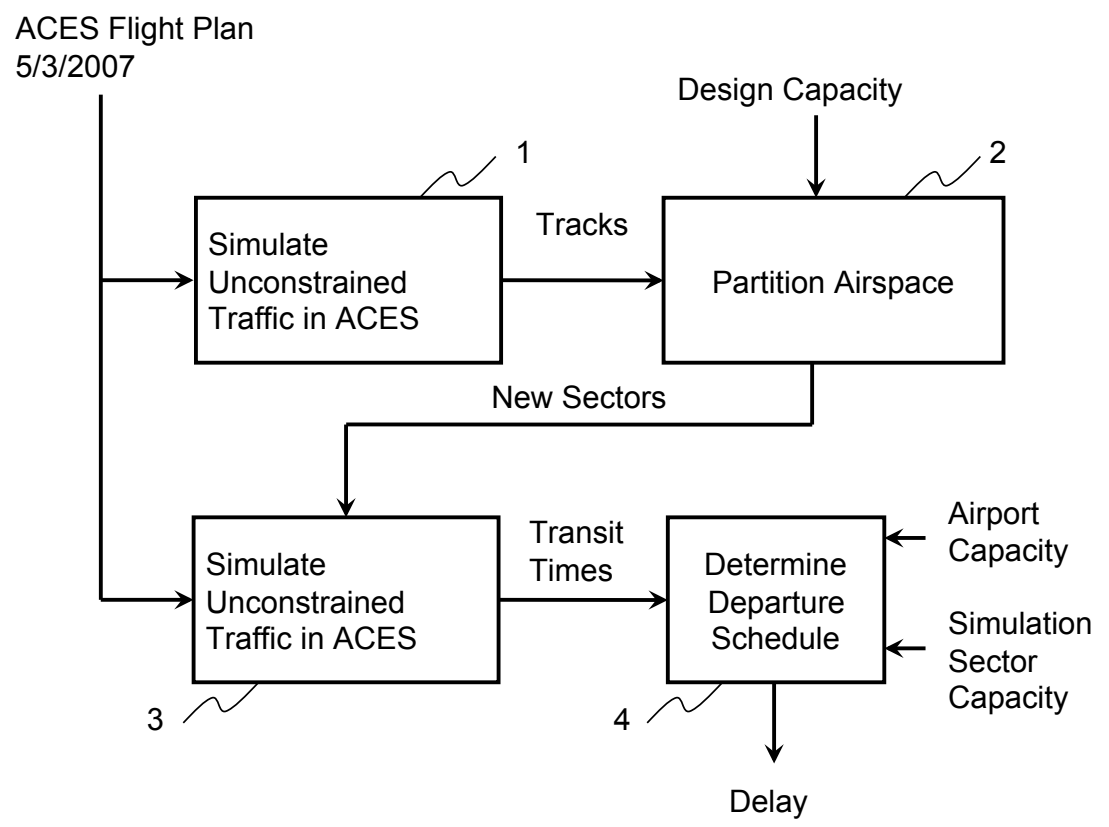

Figure 4. Two-step hierarchical method.

out to an output database.

Aircraft position data from the output database are then input to the Voronoi-diagram-based partitioning method to design sectors in the 20 centers in Block 2. A design capacity, as distinguished from the simulation sector capacity described below, of a sector is provided as an input to the airspace partitioning algorithm. This design capacity is a target capacity such that a sector being designed will not go over capacity in aircraft peak-count. The new set of sectors generated by the algorithm are written out to a file. This completes the first step of the two-step hierarchical method. Fig. 5 shows the number of sectors designed with 10 different design capacities from 9 to 30. For comparison, this figure also shows the actual average sector capacity of 17 for 373 sectors at altitude 24,000 feet and above.

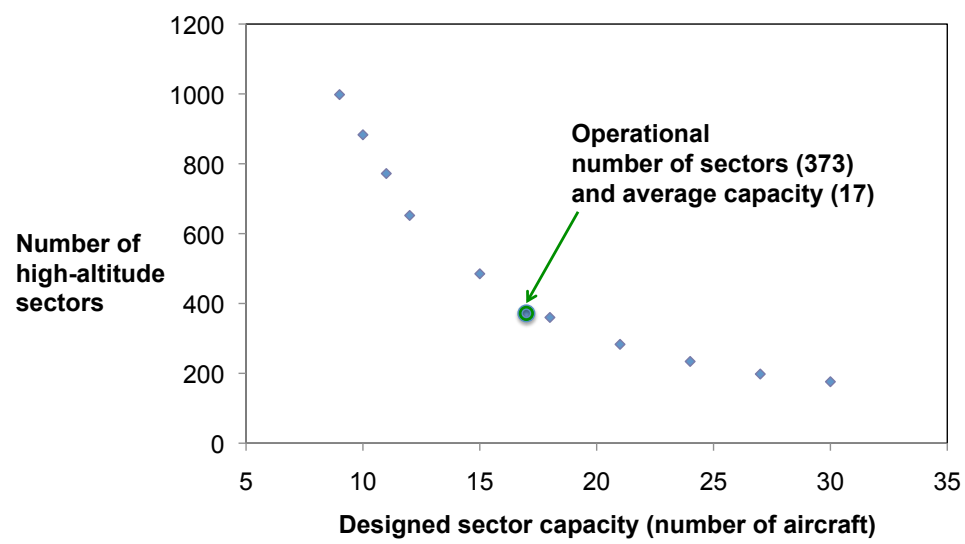

Figure 5. Number of sectors as a function of design capacities.

The second step of the hierarchical method is implemented by Block 3 in which the new sectors are input along with the flight plan file to simulate air traffic without airport and sector capacity constraints. Aircraft position and sector occupancy data output from ACES are processed to determine times of entry and exit at sectors along the flightplan of every flight. Time of arrival of each flight at the destination airport is also computed. These data are provided to the departure scheduler. The departure scheduler also needs airport and sector capacities. Airport arrival and departure capacities are specified as number of aircraft arrivals and departures per hour. Sector capacity is specified as the maximum number of aircraft allowed in a sector at any given time. The departure scheduler computes a new departure schedule for flights that includes delays needed for ensuring that the specified airport and airspace capacities are not 
exceeded.

The simulation sector-capacities specified in Block 4 are capacities for traffic flow management. They refer to the maximum number of aircraft the traffic flow management will allow during a simulation.On the other hand, the design capacity in Block 2 is a target value during sector partitioning such that the sector will accommodate aircraft count up to the design capacity. In this study, we set the simulated capacities to the corresponding designed values, except in one special case. For example, the sectors designed for a capacity of 30 was used for traffic flow management to constrain air traffic to 30 aircraft peak-count. For the special case, the sectors designed for a capacity of 21 was also used for traffic flow management to constrain air traffic to 18 aircraft peak-count to see the tradeoff of the amount of increased delay and reduced number of sectors.

\section{Results}

The interaction between the number of sectors and the delay needed for complying with the capacity constraints studied using the two-step hierarchical method described in Fig. 4 is discussed in Section IV.A. Results are also presented to show the impact of 15 and 20 percent traffic growth over the current traffic levels with assumed airport capacities in years 2018 and 2025 on delays in Section IV.B.

\section{A. Interaction between Number of Sectors and Delay}

This section describes the input scenario and assumption for the interaction part of the study, how the approach described earlier is applied, and the result. The result is presented first with a detail look at a specific partition of 283 sectors designed for a capacity of 21 . Next the interaction revealed by the simulation results of the entire 10 sector partitions from 176 sectors to 998 sectors are discussed.

Recall the approach outlined in Fig. 4 was started by initially preparing the flight plan input. This step involves converting 28 hours of May 3, 2007, air traffic data (with a four-hour data from the next day) in the recorded ASDI file to flight plan file needed for ACES simulations. All international flights and those within the same terminal radar approach control (TRACON) area were discarded. Block 1 of Fig. 4 was then run to generate aircraft position data for the 50,042 flights in the data set. Sector design capacities of 9 through 30 aircraft were then specified in Block 2 to design high-altitude sectors starting at 24,000 feet altitude in the 20 centers for each design capacity. This resulted in 10 different sector partitions with 176 to 998 sectors, some of which are shown in Fig. 5. The high-altitude sectors in each center were then placed above a single infinite capacity low-altitude sector covering the center. Each of these designs were then used with the flight plan input file in Block 3 to compute the data needed for the departure scheduler. These data were then used in Block 4 to generate departure schedules with delays for the 50,042 flights for meeting the airport and airspace capacity constraints. Arrival and departure capacities of 73 major U. S. airports on May 3rd reported in the Federal Aviation Administration's (FAA) Aviation System Performance Metrics (ASPM) database were specified in Block 4. Sector capacities between 9 and 30 aircraft were used in Block 4 to generate the delays for the study. The initial scheduled departure times in the flight plan file were replaced with the departure schedules generated in Block 4. These files were then run with the input sector partitions in Block 3 to verify that the airport and sector capacities are not exceeded.

For the result of one specific partition, consider the one corresponding to the Voronoi design capacity of 21 aircraft with simulation sector capacity of 21 aircraft. One would expect a small amount of delay with a simulation sector capacity of 21 aircraft because the same capacity was used in the design. Delay statistics for this simulation are given in Table 2. The fact that the departure scheduler can delay some flights by 6 hours, which is unreasonable, is apparent in the table. Airlines are expected to cancel flights with large delays therefore such delays should not be observed in the real air traffic data. The distribution of delays of the 10,015 flights with 283 sectors associated with this specific partition and simulation sector capacity of 21 aircraft is shown in Fig. 6. This figure suggests the limitations of the simple FCFS departure scheduling algorithm. A better algorithm that not only considers flights that were scheduled first but also flights that have been delayed the most in building the departure schedule might be able to lower the variance for a more equitable distribution of delays to flights.

In addition, we also used a reduced simulation sector-capacity for this partition to see whether it is possible to have less number of sectors. If possible, we can potentially tradeoff an increased in delay for a smaller number of sectors. The result shown in Fig. 10 indicates that an increased delay of around 15 seconds (2\%) can be traded for around $21 \%$ decrease of the number of sectors (360 to 283). Next, result of the entire 10 sector partitions is described.

The same procedure described in this example was repeated for the other partitions. Average delay resulting from the simulations are provided in Fig. 10. The point marked with a diamond corresponds to a simulation result of setting 
Table 2. Delay statistics when sector capacity is 21 .

\begin{tabular}{lr}
\hline \hline Metric & Voronoi 21 with capacity 21 \\
\hline Total \# Flights & 50,042 \\
\# flights with $\geq 15$ minute delay & 10,015 \\
Mean delay (minutes) & 11 \\
Median delay (minutes) & 2.5 \\
Standard deviation (minutes) & 21 \\
Maximum delay (minutes) & 398 \\
Total delay (hours) & 9,396 \\
\hline
\end{tabular}

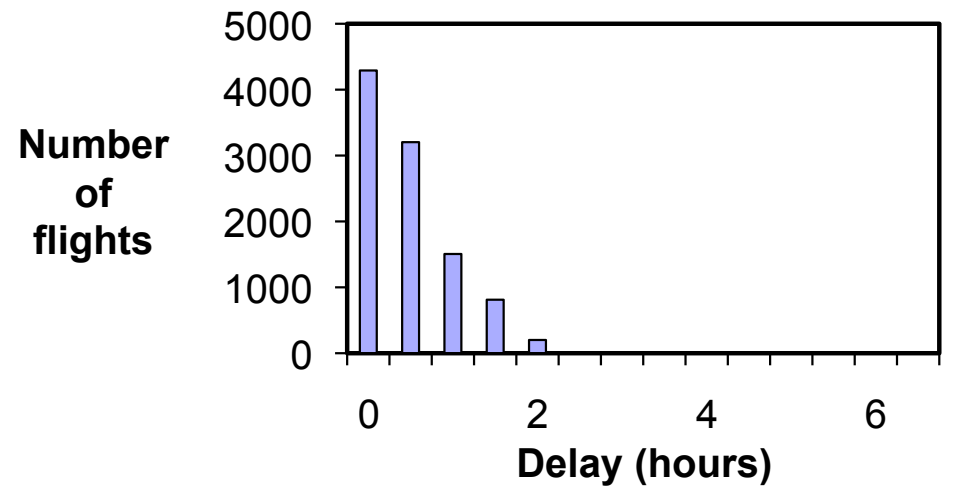

Figure 6. Delay histogram of flights delayed by $\geq 15$ minutes with design capacity of 21 aircraft and simulation sector capacity of 21 aircraft. 
the sector capacity to 9 aircraft with a 998 -sector partition. The next point marked with a square for a sector capacity of 10 aircraft with a 883-sector partition. The rest of the points are similar; the last point is for the sector capacity of 30 aircraft with a 176-sector partition. The figure shows that all delays are approximately 11 minutes of average per flight delay. This delay is solely due to airport capacity constraints. For comparison, the actual operational daily average, total system delay was around 31 minutes per delayed flight for May 3, 2007, as reported by the FAA Operations Network database. ${ }^{\circ}$

Although these sector partitions were generated by an algorithm and their viability for use in actual air traffic operations is questionable, 360 sectors compare favorably with around 373 actual sectors with an average capacity of 17 aircraft used operationally, described in FAA's May 2007 Enhanced Traffic Management System (ETMS) sector adaptation data. The 373 actual sectors are those that the simulated traffic flew through. The interaction between delay and number of sectors suggests that it might be possible to re-partition the airspace to regain loss of airspace capacities during weather-impacted days without significant delay cost around this operating region.

Figure 10 shows that all delays are mostly due to airport constraints. For the sector partition designed for the capacity of 21 and current traffic, delay increases slightly with a reduced sector capacity to 18 . The next section shows that the same effect of delay increase is observed with increasing traffic for a chosen sector partition.

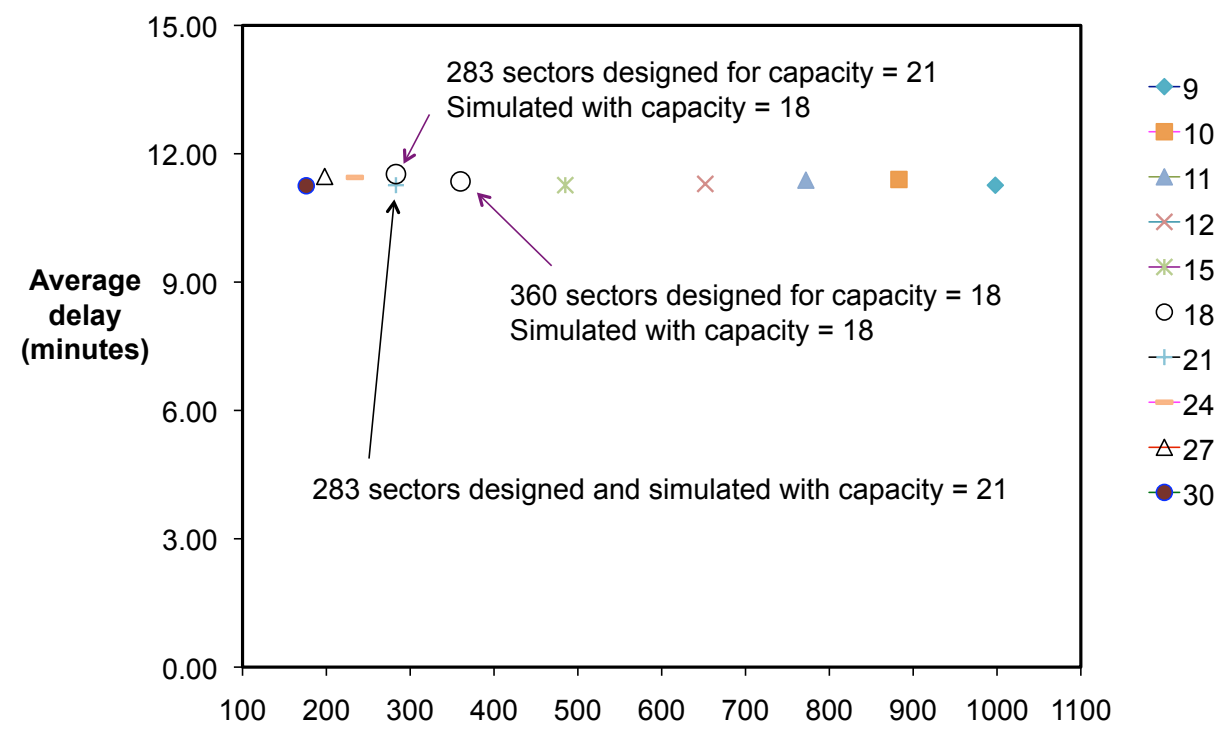

Number of high-altitude sectors

Figure 7. Interaction between delay and sector partitions.

\section{B. Traffic Growth Impact on Delays}

This section describes the future input scenarios, how the approach described in Fig. 4 is applied, and the result. The first result involves a traffic increase assumption with current and forecasted airport capacities. It focuses on the behavior of delays assuming better future technology enabling higher airspace capacities. The second result also involves future traffic, current and forecasted airport capacities. It focuses on the behavior of delays due to traffic increases.

The future input scenarios consist of $15 \%$ and $20 \%$ increase in traffic above 2007 levels, as well as the forecasted airport capacities in years 2018 and 2025. Instead of using the flight plan file corresponding to May 3, 2007 ASDI data, the traffic data in the flight plan file was grown by $15 \%$ and $20 \%$ using the AvDemand Tool described in Ref. 27. Scheduled departure times and routes of the May 3, 2007 baseline traffic were preserved in the grown traffic data

\footnotetext{
$\S$ http://aspm.faa.gov/opsnet/sys/Delays.asp
} 
sets. Capacities of the 71 major U. S. airports were increased on an average by $7 \%$ for year 2018 and by $22 \%$ for year 2025. The range of growth spanned from no increase at Newark Liberty International Airport to $45 \%$ increase at Seattle Tacoma International Airport in 2018, and from no increase at Palm Springs International Airport to 84\% increase at Seattle Tacoma International Airport in 2025. These growth rates were based on analysis of FAA data, including Terminal Area Forecast, ${ }^{28}$ OEP35 2004/2006 Baselines and percent capacity increase estimates, FAA AC 150-5060-5, ${ }^{29}$ FAA 2004 Benchmark $^{30}$ and FAA 2007 FACT-2, ${ }^{31}$ their Next Generation Air Transportation System implementation plan of current, 2018, and 2025 time frames. The actual airport arrival and departure capacities on May 3, 2007 were multiplied with these growth factors and input to Block 4 in Fig. 4 to generate the delays.

Figure 8 shows the average TFM delay incurred per flight with $15 \%$ traffic growth as the sector capacity of 360 sectors, designed for capacity of 18 aircraft with the baseline (one-times) traffic, was increased from 18 aircraft to 24 aircraft. The line marked with diamonds shows delays for the baseline airport capacities. The lines marked with squares and triangles show the results with 2018 and 2025 airport capacities, respectively. As expected, the delays decrease with increasing airport capacities. Increase in sector capacity from 18 to 24 aircraft has virtually no impact on reducing the delays. This indicates that delays will continue to be driven mostly by airport capacities. Results indicate that $15 \%$ growth will result in about 11 minutes of delay per flight with 2025 airport capacities, which is the same as the delays with current traffic with current airport capacities.

Figure 9 shows the increase in average flight delays as a function of traffic growth and the decrease of the delays as a function of airport capacities. The sector partition with 360 sectors with a capacity of 18 aircraft was used for generating these results. The graphs marked with circles, squares, and triangles correspond to the baseline airport capacities, 2018 airport capacities, and 2025 airport capacities, respectively. The trends shown in the graphs agree qualitatively with those in Fig. 3 of Ref. 32.

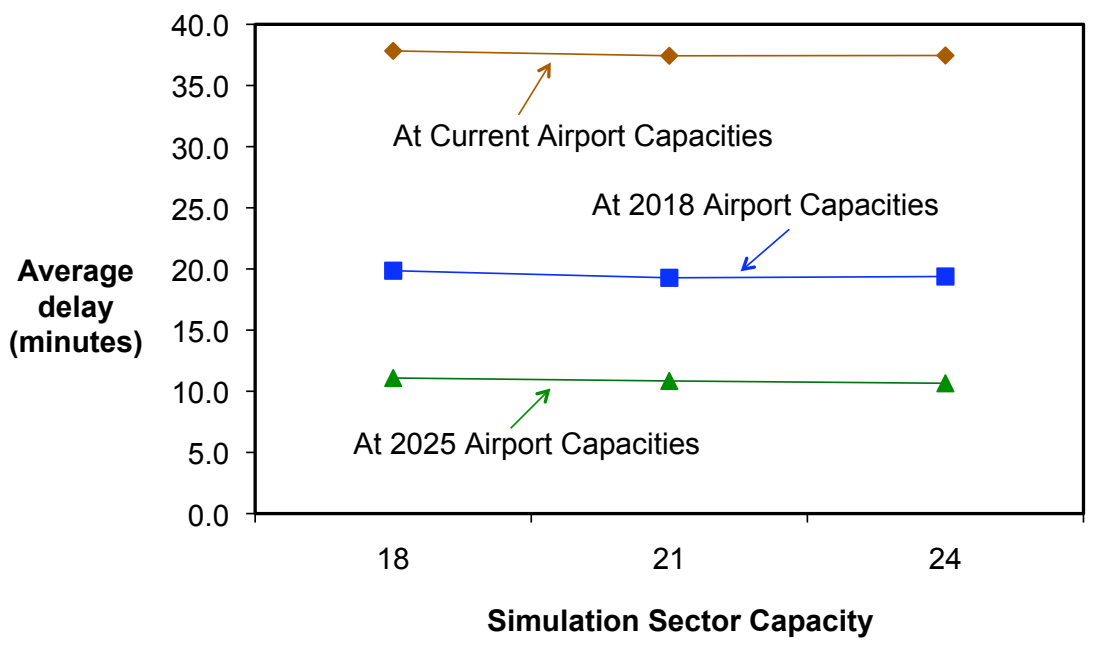

Figure 8. Delays as a function of increasing airport and sector capacities. 


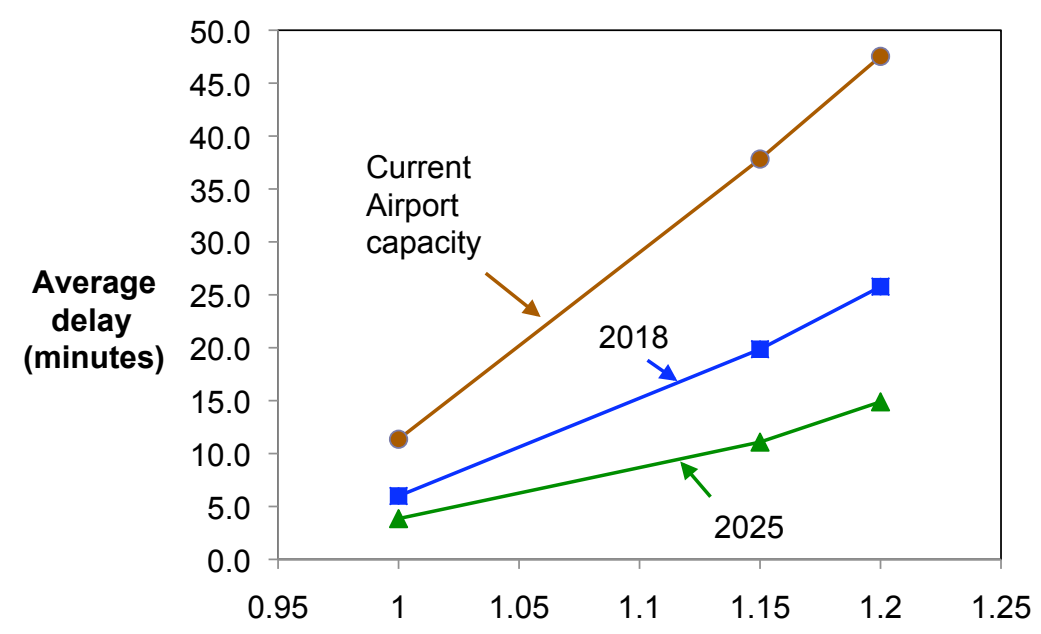

Traffic Demand at $1 \mathrm{x}, 1.15 \mathrm{x}$, and $1.2 \mathrm{x}$

Figure 9. Delays as a function of increasing traffic growth and forecasted airport capacities.

\section{Conclusions}

In the current air traffic system, techniques such as, delaying flights on the ground and metering in the airspace, are commonly employed to constrain the demand to comply with airport and airspace capacity constraints. In this paper, the interaction between partitioning of clear-weather day airspace for distributing capacity and delays caused by traffic flow management has been examined for current and future traffic demand and airport capacity scenarios. The approach for studying the interaction consisted of simulating air traffic using the Airspace Concept Evaluation System, designing sectors with the Voronoi-based method, and computing delays with the first-come first-served departure scheduler based on airspace and airport capacity constraints.

To study the interaction between airspace partitions and TFM delay, 10 sector partitions were created with capacities of 9 to 30 aircraft. These partitions were then used during air traffic simulations to generate transit-time data for the departure scheduler. Delays were then computed using the departure scheduler with specified simulation sector capacities. Results show that the delays are driven by airport arrival and departure capacity constraints. Results also show that delays caused by airspace capacity constraints can be eliminated by partitioning the airspace and that the number of sectors can be reduced without significantly increase the average delay. This suggests that it might be possible to re-partition the airspace to regain loss of airspace capacities during weather-impacted days. In follow-up studies, interaction between airspace partitions and TFM delay in the presence of weather will be explored to determine if there are some local benefits for flights in the weather-impacted regions.

Delays resulting from traffic growth of $15 \%$ and $20 \%$ with current, forecasted 2018 and 2025 airport capacities were examined. Results show that a $20 \%$ traffic growth would increase the per flight delay from 11.4 minutes to 47.5 minutes, which a four-fold increase. With 2018 and 2025 airport capacities, the delay per flight would be reduced to 25.8 minutes and 14.4 minutes, respectively. Analysis of $15 \%$ traffic growth with increasing sector capacities showed that only a marginal reduction in delays can be expected; airport capacity constraints will continue to remain the driving factor.

\section{Acknowledgments}

The authors thank Charlene Cayabyab for configuring and running the simulations. Discussions with Dr. Allen Goldberg led to the development of the FCFS departure scheduler. Michael Downs provided the future traffic growth and airport capacities in years 2018 and 2025. He also created an integrated version of the Airspace Concept Evaluation System (ACES) in which the FCFS scheduler is used with airborne delay maneuvers to comply with airport and airspace capacity constraints. This version of ACES provides additional capabilities to conduct follow-up studies. 


\section{Appendix}

The complete set of test cases, including other reduced-capacity cases, and the entire result set are presented in this section for completeness. The test matrix consisting of combinations of capacities used for generating sectors and capacities specified for generating departure schedules is shown in Table 3. The result in average delay resulting from the entire test cases is provided in Fig. 10.

Table 3. Test matrix.

\begin{tabular}{|c|c|c|c|c|c|c|c|c|c|c|}
\hline $\begin{array}{l}\text { Voronoi } \\
\text { Design }\end{array}$ & \multicolumn{10}{|c|}{ Sector Capacity for TFM } \\
\hline 30 & 9 & 10 & 11 & 12 & 15 & 18 & 21 & 24 & 27 & 30 \\
\hline 27 & 9 & 10 & 11 & 12 & 15 & 18 & 21 & 24 & 27 & $x$ \\
\hline 24 & 9 & 10 & 11 & 12 & 15 & 18 & 21 & 24 & $x$ & $x$ \\
\hline 21 & 9 & 10 & 11 & 12 & 15 & 18 & 21 & $x$ & $x$ & $x$ \\
\hline 18 & 9 & 10 & 11 & 12 & 15 & 18 & $x$ & $x$ & $x$ & $x$ \\
\hline 15 & 9 & 10 & 11 & 12 & 15 & $x$ & $x$ & $x$ & $x$ & $x$ \\
\hline 12 & 9 & 10 & 11 & 12 & $X$ & $x$ & $X$ & $x$ & $x$ & $x$ \\
\hline 11 & 9 & 10 & 11 & $x$ & $X$ & $x$ & $x$ & $x$ & $x$ & $x$ \\
\hline 10 & 9 & 10 & $x$ & $x$ & $x$ & $x$ & $x$ & $x$ & $x$ & $x$ \\
\hline 9 & 9 & $X$ & $x$ & $x$ & $x$ & $x$ & $X$ & $x$ & $X$ & $x$ \\
\hline
\end{tabular}

The graph marked with diamonds corresponds to a simulation sector capacity of 9 aircraft with the 10 sector partitions. The next graph marked with squares for a sector capacity of 10 aircraft has 9 data points. The rest of the graphs are similar; the last graph for the sector capacity of 30 aircraft has a single data point. The figure shows that delays increase for designs with fewer sectors and lower sector capacity values. The set of graphs provides a basis for selecting a design with fewer sectors at the cost of increased delay. This represents a tradeoff between the cost to the service provider in terms of resources needed for operating each sector and the cost to the airlines in terms of delay caused by TFM. If sector controllers can handle 18 aircraft in 360 sectors, flights would be delayed by 11.4 minutes on average. On the other hand, if the controllers can handle 18 aircraft in 234 sectors, flights would be delayed by 12.8 minutes.

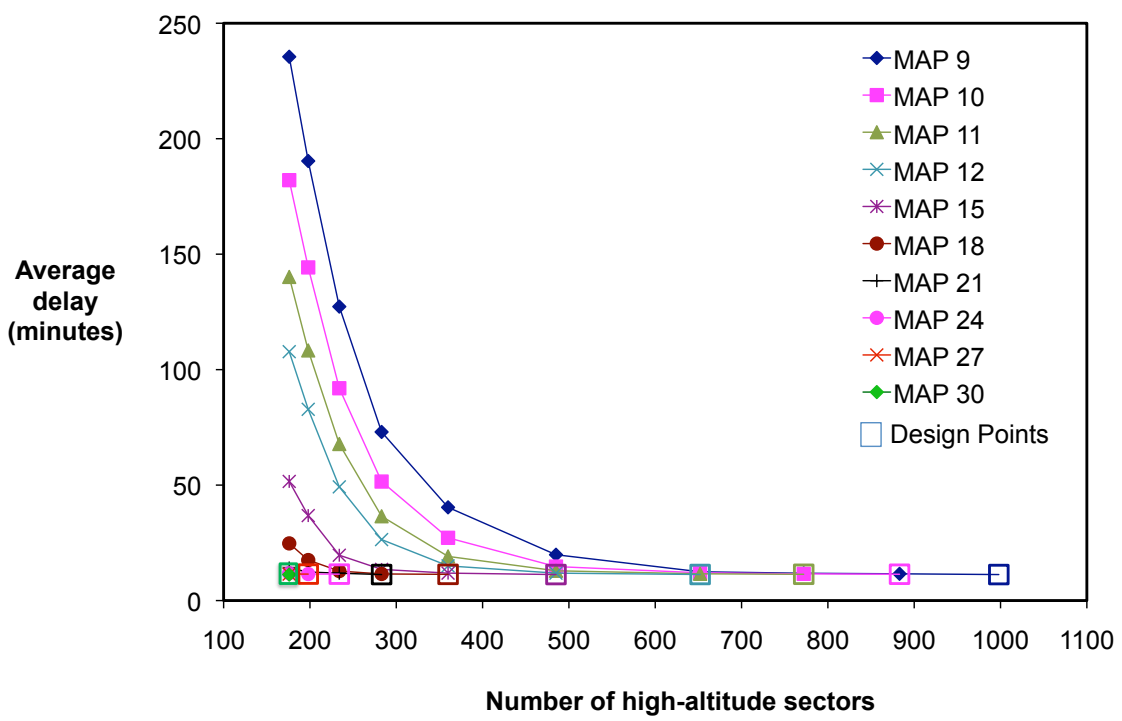

Figure 10. Tradeoff between delay and sector partitions. Simulation sector capacity is indicated as MAP. 


\section{References}

${ }^{1}$ Sridhar, B., Chatterji, G. B., Grabbe, S., and Sheth, K., "Integration of Traffic Flow Management Decisions," AIAA Guidance, Navigation and Control Conference, Monterey, CA, Aug. 5-8, 2002.

${ }^{2}$ Sridhar, B., Soni, T., Sheth, K., and Chatterji, G., "An Aggregate Flow Model for Air Traffic Management," AIAA Guidance, Navigation and Control Conference and Exhibit, Providence, RI, Aug. 16-19, 2004.

${ }^{3}$ Grabbe, S. and Sridhar, B., "Congestion Management with an Aggregate Flow Model," AIAA Guidance, Navigation and Control Conference and Exhibit, San Francisco, CA, Aug. 15-18, 2005.

${ }^{4}$ Sridhar, B., Grabbe, S., and Mukherjee, A., "Modeling and Optimization in Traffic Flow Management," Vol. 96, 2008, pp. $2060-2080$.

${ }^{5}$ Grabbe, S., Sridhar, B., and Mukherjee, A., "Integrated Traffic Flow Management Decision Making," AIAA Guidance, Navigation and Control Conference, Chicago, IL, Aug. 10-13, 2009.

${ }^{6}$ Grabbe, S., Sridhar, B., and Mukherjee, A., "Sequential Traffic Flow Optimization with Tactical Flight Control Heuristics," Vol. 32, 2009, pp. 810-820, also AIAA Paper 08-6823, 18-21 Aug. 2008.

${ }^{7}$ Sun, D., Sridhar, B., and Grabbe, S., "Disaggregation Method for an Aggregate Traffic Flow Management Model," Vol. 33, 2010, pp. 666-676.

${ }^{8}$ Kopardekar, P., Bilimoria, K., and Sridhar, B., "Initial Concepts for Dynamic Airspace Configuration," 7th AIAA Aviation Technology, Integration and Operations Conference (ATIO), Belfast, Northern Ireland, Sep. 18-20, 2007.

${ }^{9}$ Yousefi, A. and Donohue, G. L., "Temporal and Spatial Distribution of Airspace Complexity for Air Traffic Controller Workload-Based Sectorization," 4th AIAA Aviation Technology, Integration and Operations Conference (ATIO), Chicago, IL, Sep. 20-22, 2004.

${ }^{10}$ Klein, A., "An Efficient Method for Airspace Analysis and Partitioning Based on Equalized Traffic Mass," 6th FAA and Eurocontrol ATM Conference, Baltimore, MD, Jun. 2005.

${ }^{11}$ Martinez, S. A., Chatterji, G. B., Sun, D., and Bayen, A. M., "A Weighted-Graph Approach for Dynamic Airspace Configuration,” AIAA Guidance, Navigation, and Control Conference and Exhibit, Hilton Head, SC, Aug. 20-23, 2007.

${ }^{12}$ Mitchell, J. S. B., Sabhnani, G., Krozel, J., Hoffman, R., and Yousefi, A., "Dynamic Airspace Configuration Management Based on Computational Geometry Techniques,” AIAA Guidance, Navigation, and Control Conference and Exhibit, Honolulu, Hawaii, Aug. 18-21, 2008.

${ }^{13}$ Drew, M., "Analysis of an Optimal Sector Design Method," 27th Digital Avionics Systems Conference, St. Paul, MN, Oct. 2008.

${ }^{14}$ Xue, M., "Airspace Sector Redesign Based on Voronoi Diagrams," AIAA Guidance, Navigation, and Control Conference, Honolulu, HI, Aug. 2008.

${ }^{15}$ Hunter, G., "Interactions between single-center dynamic resectorization and NextGen TFM," IEEE/AIAA 28th Digital Avionics Systems Conference, 23 - 29 Oct. 2009.

${ }^{16}$ Hackney, B., Hoffman, R., Khorrami, B., Kicinger, R., Kostitsyna, I., Lowther, M., Mitchell, J., Sabhnani, G., Viswanathan, R., and Yousefi, A., "Preliminary Benefit Assessment of Airspace Allocation Schemes Dynamic Airspace Configuration," Metron Aviation Report, NASA Control No. 32N0809-017, Sept. 2009.

17 "Facility Operation and Administration," Order JO 7210.3V, Federal Aviation Administration, Feb. 2008.

${ }^{18}$ Bertsimas, D. J., Lulli, G., and Odoni, A., "The Air Traffic Flow Management Problem:An Integer Optimization Approach,” 13th International Conference, IPCO, 26-28 May 2008.

${ }^{19}$ Xue, M., "Three Dimensional Sector Design with Optimal Number of Sectors," AIAA Guidance, Navigation and Control Conference, Toronto, Ontario, Canada, Aug. 2-5, 2010.

20 “Airport Surface Delays and Causes, A Preliminary Analysis," Nasa contractor report 201721, National Aeronautics and Space Administration, Aug. 1997.

21 "National Airspace System, DOT and FAA Actions Will Likely Have a Limited Effect on Reducing Delays during Summer 2008 Travel Season," GAO-08-934T, United States Government Accountability Office, July 2008.

22 "U.S./Europe Comparison of ATM-related Operational Performance," 2009-AJG-333, EUROCONTROL and Federal Aviation Administration, Oct. 2009.

${ }^{23}$ Meyn, L., Windhorst, R., Roth, K., Drei, D. V., Kubat, G., Manikonda, V., Roney, S., Hunter, G., Huang, A., and Couluris, G., "Build 4 of the Airspace Concept Evaluation System," AIAA Modeling and Simulation Technologies Conference and Exhibit, Keystone, Colorado, 21-24 Aug. 2006.

24"User Manual for the Base of Aircraft Data (BADA) Revision 3.6," EEC Note No. 10/04, EUROCONTROL Experimental Centre, July 2004.

${ }^{25}$ Zelinski, S. J., "Validating the Airspace Concept Evaluation System Using Real World Data," AIAA Modeling and Simulation Technologies Conference and Exhibit, San Francisco, CA, Aug. 15-18, 2005.

${ }^{26}$ Zelinski, S. J. and Meyn, L., "Validating the Airspace Concept Evaluation System for Different Weather Days," AIAA Modeling and Simulation Technologies Conference and Exhibit, Keystone, CO, Aug. 21-24, 2006.

${ }^{27}$ Huang, A. S., Schleicher, D., and Hunter, G., "Future Flight Demand Generation Tool," AIAA Aviation Technology, Integration and Operations Forum (ATIO), Chicago, Illinois, Sep. 20-22, 2004.

28 "Terminal Area Forecast," FAA-APO-00-7, Federal Aviation Administration, Dec. 2000.

29 “Airport Capacity and Delay," AC: 150/5060-5, Federal Aviation Administration, Sept. 1983.

30 “Airport Capacity Benchmark Report 2004,” Report, Federal Aviation Administration, Sept. 2004.

31 "Capacity Needs in the National Airspace System 2007-2025," HQ-009107.INDD, Federal Aviation Administration, May 2007.

${ }^{32}$ Gawdiak, Y., Carr, G., and Hasan, S., "JPDO Case Study of NextGen High Density Operations," 9th AIAA Aviation Technology, Integration, and Operations Conference (ATIO), Hilton Head, South Carolina, 21-23 Sep. 2009. 